Research report

\title{
Egocentric and object-based transformations in the laterality judgement of human and animal faces and of non-corporeal objects
}

\author{
Silvio Ionta ${ }^{\mathrm{a}, \mathrm{b}, *}$, Alissa D. Fourkas ${ }^{\mathrm{a}, 1}$, Salvatore M. Aglioti ${ }^{\mathrm{a}, \mathrm{b}, * *}$ \\ a Department of Psychology, University of Rome “La Sapienza”, via dei Marsi 78, 00185 Roma, Italy \\ b IRCCS “Fondazione Santa Lucia”, via Ardeatina 306, 00179 Rome, Italy
}

\section{A R T I C L E I N F O}

\section{Article history:}

Received 19 August 2009

Received in revised form 21 October 2009

Accepted 26 October 2009

Available online 4 November 2009

\section{Keywords:}

Mental rotation

Mental imagery

Postural constraints

Mental spatial transformations

Face inversion effect

Body representation

\begin{abstract}
A B S T R A C T
Mental rotation of body parts is influenced by specific sensory-motor information, and may be performed using an egocentric (subject-based) or an object-based mental transformation. Neurologically healthy volunteers were asked to verbally judge the laterality of visually presented human face, owl face and front of a car with a black patch over one eye/headlight, presented in one of eight orientations. Subjects may or may not have their head held in a head brace. The transformation used to solve the task was assessed with a questionnaire. Response times were non-monotonical at $180^{\circ}$ for the object-based group, but not for the group using egocentric transformation. Having head movement constrained by the use of a head brace ("fixed") or not ("moving") did not influence performance. Within the two groups, no differences were found between the three types of stimuli. Hence, the response profile for mental rotation of human faces and face-like stimuli depended on the type of mental spatial transformation used to solve the task, independently from the possibility to move the head and from the kind of stimuli processed.
\end{abstract}

(c) 2009 Elsevier B.V. All rights reserved.

\section{Introduction}

Mental imagery can be defined as the activation of an inner representation that causes an almost-perceptive experience in absence of an appropriate sensorial stimulation. It is used to transform and manipulate mental images and to mentally simulate actions. The simulation of a movement requires an amount of time that is proportional to the time needed to actually perform it $[32,41]$, determines a specific facilitation on motor potential evoked by TMS [16] and activates a neural network partially overlapping with that involved in the movement planning [13] and execution [23,32]. Moreover, neurovegetative activation during physical effort is proportional to that measured during the mental simulation of the same task [12] and different cerebral lesions may determine selective and dissociable imagery deficits [41]. Given the stability of temporal and kinematic characteristics of real and sim-

\footnotetext{
* Corresponding author at: Laboratory of Cognitive Neuroscience, Brain-Mind Institute, Ecole Polytechnique Fédérale de Lausanne (EPFL), Station 19, 1015 Lausanne, Switzerland. Tel.: +4121 6931775; fax: +41216931770.

** Corresponding author at: Department of Psychology, University of Rome "La Sapienza", via dei Marsi 78, 00185 Roma, Italy. Tel.: +39 06 49917601; fax: +3906 49917635.

E-mail addresses: silvio.ionta@epfl.ch (S. Ionta), salvatoremaria.aglioti@uniroma1.it (S.M. Aglioti).

${ }^{1}$ Now at: Human Cortical Physiology Section, National Institute of Neurological Disorders and Stroke, 10 Center Drive, Bethesda, MD 20892-1430, United States.
}

ulated movements, it is reasonable that the same properties guide both execution and simulation of the same movement [1,13,31]. In this way mental rotation (a class of mental imagery tasks) can be considered a good tool to study motor representations.

The effect of postural signals can produce an effect very specific to the mentally manipulated body part $[11,26,40]$. For example, if people are requested to judge the laterality of hands and feet pictures, while varying the posture of their own hands but not of their feet, then response time varies for hands' judgement but not for feet [25]. Moreover, mental rotation of body parts seems to be influenced by both central and peripheral factors. Indeed it can be impaired by transcranial magnetic stimulation applied over the primary motor cortex of healthy subjects [21], by cortical [43] and subcortical [24] lesions, as well as in patients with upper limb amputation [29], locked-in syndrome [6], chronic upper limb pain [37] and Parkinson's disease [15]. In addition, patients with idiopathic cervical dystonia show an impairment in the mental rotation of body parts but not for non-body parts, such as cars [18], suggesting the dissociation between the mental manipulation of body and non-body parts [6].

The present study tests the effect of constraining head movement during mental rotation. This behaviour is often observed in participants taking part in experiments and the potentially specific effect of such a behaviour on different classes of stimuli (human, animal, and inanimate). In the present study participants were asked to judge the laterality of different types of stimuli (human face, front of a car, and owl face) in two different conditions: 


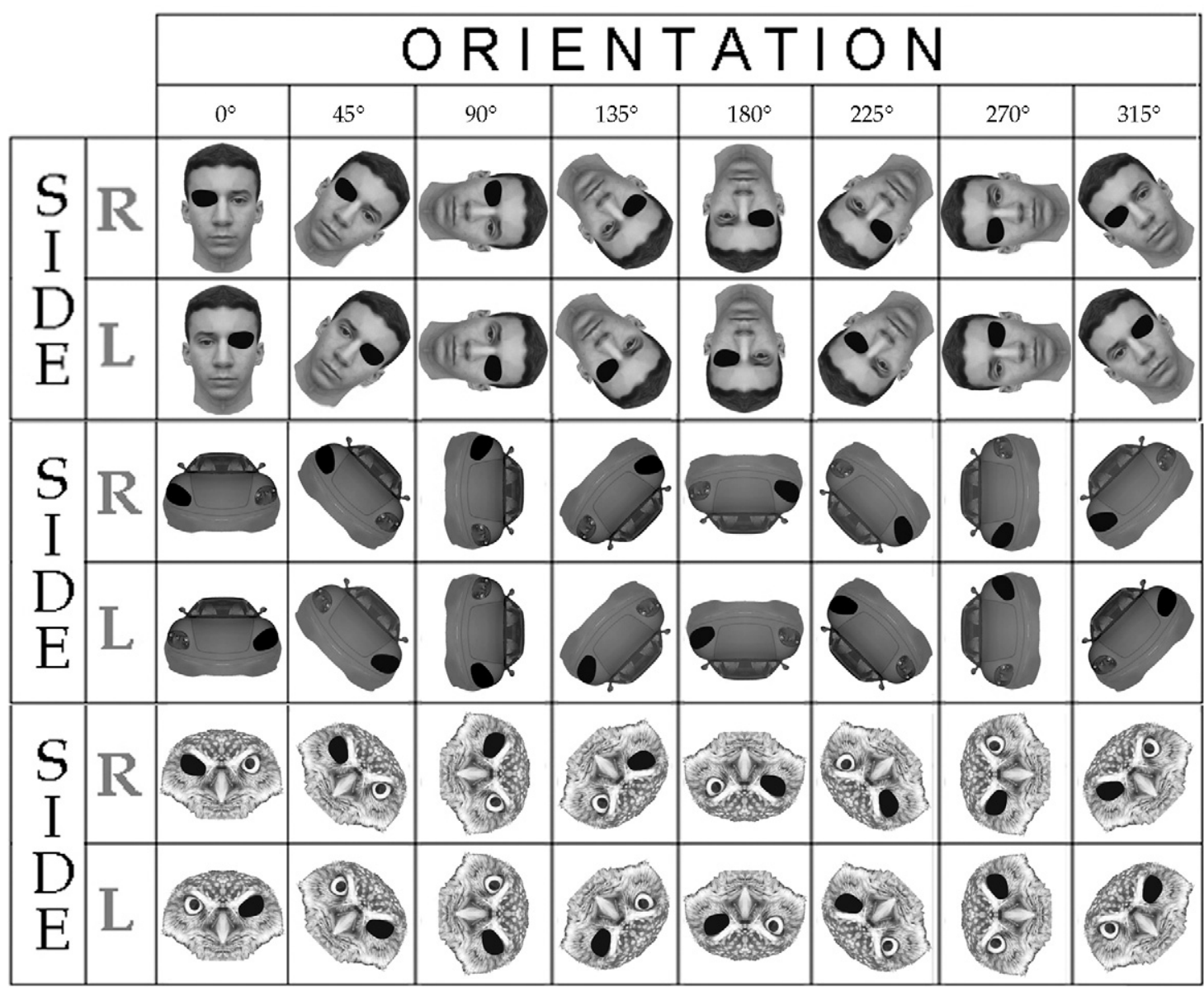

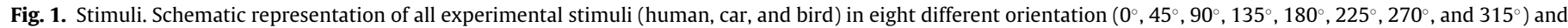
two lateralities ( $\mathrm{R}=$ right and $\mathrm{L}=\mathrm{left}$ ).

holding, or not, the head in a head brace. After each session, they were also asked to complete a questionnaire on the kind of mental transformation they used to perform the task. If after the adoption of one kind of mental transformation, objects are treated in the same way regardless to their nature, there should be no differences in response times or accuracy between the different stimuli.

\section{Materials and methods}

\subsection{Participants}

Twenty-two participants (seven females) aged 19-33 ( $M=25.8$ years, SD $=3.2)$ and all with a college undergraduate level of education, took a part in the experiment. All were right-handed according to an handedness inventory [2]. Ethical approval was obtained by the local ethical committee and written informed consent obtained prior to participation. The study was carried out in accordance with the ethical standards of the 1964 Declaration of Helsinki.

\subsection{Stimuli}

The stimuli, illustrated in Fig. 1, were pictures of a human face, an owl face and the front of a car, oriented at the upright and in one of seven clockwise orientations from the upright $\left(0^{\circ}, 45^{\circ}, 90^{\circ}, 135^{\circ}, 180^{\circ}, 225^{\circ}, 270^{\circ}\right.$, and $\left.315^{\circ}\right)$ presented one at a time in the centre of a computer monitor. The upright view was defined as nose line pointing upwards at $0^{\circ}$ of rotation. A black patch was applied over one eye (or one headlight) of each stimulus. The stimuli covered a visual angle of $11.4^{\circ}$ viewed from a distance of $60 \mathrm{~cm}$.

\subsection{Procedure}

The experimental session consisted of six randomly presented blocks. Two blocks contained 96 face pictures, two contained 96 owl pictures and two contained 96 car pictures. Each orientation was randomly depicted 12 times in each block, with the same orientation presented no more than twice in sequence. The two blocks within each stimulus type varied in terms of head constraint conditions. As depicted in Fig. 2, in one condition the participants' head was constrained by use of a head brace (fixed) and in the other condition the head was free to move (moving). The order of head constraint and stimuli type was counterbalanced across subjects.

Participants sat on a chair in front of a computer with the monitor (Apple ColorSync) positioned at $60 \mathrm{~cm}$ from the participants' eyes. Stimulus presentation was controlled with E-Prime, Beta5 (PST company). Each trial began with a fixation cross on the monitor followed by a stimulus $1000 \mathrm{~ms}$ later. Each stimulus remained visible on the screen until the response was given. Participants verbally judged, as quickly and accurately as possible, the laterality of the patch (left or right) on each stimulus. Written instructions were provided for each condition, for example: "In a few moments, photographs will be presented depicting a face with one eye covered with a black patch. The face will be rotated in different orientations on different trials. Your task is to verbally report which eye of the photographed face is covered by the patch (its left or its right) as quickly and accurately as possible. Please maintain a stable and fixed body posture, with your eyes on the fixation point $(+)$ ". Response time was automatically recorded by a microphone positioned $25 \mathrm{~cm}$ in front of the participant and connected to the computer. Response accuracy was manually entered into the computer by the experimenter and stored for off-line analysis. A semi-structured questionnaire, reproduced in Supplementary materials, was administered after each block to assess the cognitive strategy used to accomplish the task (i.e., imagery strategy).

\subsection{Data handling of questionnaire}

Nine participants [aged 19-31 ( $M=25.5$ years, $S D=4$; four females $)$ ] described mentally rotating all the stimuli until they were in an easier orientation to judge (object-based transformation). Nine other participants [aged 23-33 ( $M=25.2$ years, $\mathrm{SD}=3$; three females)] described spontaneous spatial transformations of their own body (egocentric transformation) from a position in front of the stimulus. Four subjects used a mixed strategy. Given the stark preference for one type of transformation in the majority of the participants we included the factor 'Transformation' in the analysis; this decision resulted in the exclusion of the four participants who flipped back and forth between transformations. 


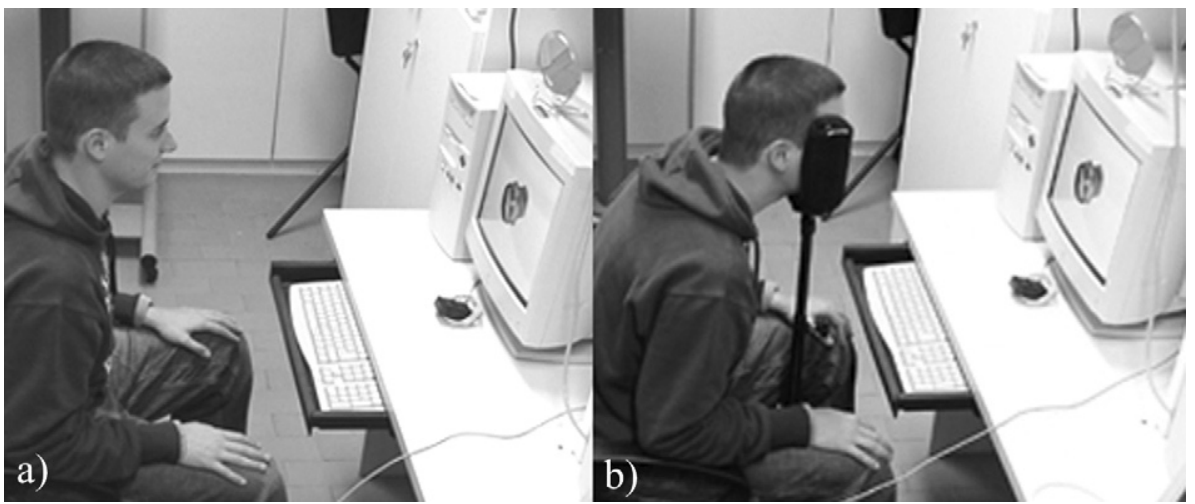

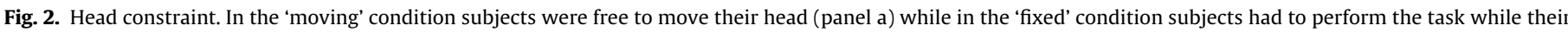
head was held in a head brace (panel b).

Qualitative responses to questions from the 18 subjects retained are summarized in Table 1 (supplementary materials), where the median responses are identified. Responses were converted from qualitative feedback (e.g. 'always true') to a 7-point numerical scale in order to perform non-parametric analyses; there were a large number of tied ranks in the data. Friedman Analysis of Variance was applied to within group data to assess whether subjects treated the stimuli differently depending on stimulus type and head constraint.

\subsection{Data handling}

Endpoint measures (response time and accuracy) were defined as in Ionta et al. [25]. Therefore response times $<500$ or $>3500$ ms were excluded from analysis, and accuracy reflects correct responses. Although incorrect responses were excluded from the analysis of response time, the number of trials lost were minimal as performance approached ceiling. Trials excluded because they were too slow or incorrect amounted to $5.6 \%$ of the total.

Response time and accuracy were each analyzed with mixed model analysis of variance (ANOVA). The two analyses included transformation (egocentric object-based), head constraint (fixed, moving), stimulus type (human, car, and owl), Stimulus laterality (left and right), and stimulus orientation $\left(0^{\circ}, 45^{\circ}, 90^{\circ}, 135^{\circ}, 180^{\circ}\right.$, $225^{\circ}, 270^{\circ}$, and $315^{\circ}$ ) as main factors, with repeated measures from the second to the fifth factor. Post hoc analyses were carried out using simple main effects (Bonferroni corrected) and the Newman-Keuls test $(p<0.05)$. The distributions tended to violate sphericity, and therefore epsilon corrections are reported as Greenhouse-Geisser (ê) if the Huyht-Feldt epsilon (ẽ) was less than 0.75 . The measure of the strength of association for main effects and interactions is reported as partial eta squared $\left(\eta_{\mathrm{p}}{ }^{2}\right)$, the proportion of variance in the dependent variable attributable to the effect. Eta squared $\left(\eta^{2}\right)$, which is dependent on the number and magnitude of other effects, is reported only for simple main effects.

\section{Results}

\subsection{Questionnaires}

Responses by subjects using an object-based strategy revealed no statistical difference across blocks for any question (all exact $p \geq 0.133$ ). Subjects using a egocentric strategy indicated a statistical difference for question (b) "in the judgement of 'right' and 'left', your response was based on the position of the patch with reference to yourself" $\chi^{2}(5)=15.28$ exact $p=0.003$ although post hoc analysis with Wilcoxon Signed Ranks found no difference between the highest (auto moving, 4.44) and lowest (face moving, 2.50) mean ranks $(p=0.156)$. The responses also indicated a difference for question $(\mathrm{g})$ "how often did you move your hand" $\chi^{2}(5)=11.23$, $p=0.036$; post hoc analysis found no significant difference between the highest (owl fixed, 4.28) and lowest (auto fixed, 2.44) mean ranks $(p=0.063)$. All other questions failed to reach significance (all $p \geq 0.067$ ). These data indicate that subject's cognitive strategy was stable across blocks.

Notably when egocentric imagers had to judge $0^{\circ}$ tilted stimuli, they performed an imagery rotation of their own body around the stimulus, insofar that they described the mental activity as a kind of "dressing the mask" of the actual stimulus that is a rotation around the $z$-axis in the ground plane [5]. Indeed, based on responses to the questionnaires the differences found between the two groups (described below) were due to the fact that, in contrast with the object-based transformation, the egocentric transformation did not involve mental rotation, regardless to the presented stimulus.

\subsection{Response time}

Response time data broken down by factor are presented in Table 2 (supplementary materials). The ANOVA showed a significant main effect of stimulus orientation $[F(7,112)=18.33 p<0.000$ with $\left.\hat{\mathrm{e}}=0.35, \eta_{\mathrm{p}}{ }^{2}=0.53\right]$ and stimulus laterality $[F(1,16)=6.63$, $\left.p=0.020, \eta_{\mathrm{p}}^{2}=0.29\right]$, both of which were superseded by significant interactions. The main effect of head constraint did not reach significance $[F(1,16)=0.39, p=0.54]$. Stimulus orientation was described by a quadratic function $\left[F(1,16)=19.63, p<0.000, \eta_{\mathrm{p}}{ }^{2}=0.55\right]$. Analysis of the main effect found that response time at $180^{\circ}(1385 \mathrm{~ms})$ was slower than at all other orientations. Response time at $135^{\circ}$ (1169 ms) was different from all the others except $225^{\circ}$ ( $1170 \mathrm{~ms}$ ); $225^{\circ}$ was different from $0^{\circ}(1085 \mathrm{~ms}), 90^{\circ}(1064 \mathrm{~ms})$ and $270^{\circ}$ $(1069 \mathrm{~ms})$. The main effect of stimulus laterality was accounted for by slower responses to left $(1149 \mathrm{~ms})$ with respect to right (1103 ms) stimuli.

Transformation interacted with stimulus orientation $\left[F(7,112)=8.87, \quad p<0.000, \quad \eta_{\mathrm{p}}^{2}=0.36\right]$, as illustrated in Fig. 3 (left panel). The simple main effect of the object-based transformation $\left[F(7,56)=18.47, p<0.000\right.$ with $\left.\hat{\mathrm{e}}=0.24, \eta^{2}=0.70\right]$ was the only component of the interaction to be significant with Bonferroni correction $(0.05 / 10=0.005)$. The simple main effect of the egocentric transformation failed to reach corrected significance $[p=0.017$ with $\hat{\mathrm{e}}=0.27]$. To simplify interpretation and facilitate comparison to previous research, we calculated the linear function from $0^{\circ}$ to $180^{\circ}$ with data equidistant from $180^{\circ}$ collapsed (i.e., $45^{\circ}$ with $315^{\circ}, 90^{\circ}$ with $270^{\circ}$, and $135^{\circ}$ with $225^{\circ}$ ). For mental rotation tasks the slope of the linear function is interpreted as reflecting the average rate of change associated with rotating an object an additional degree (e.g. from $29^{\circ}$ to $30^{\circ}$ ) and the intercept reflects contributions of non-rotational (e.g. preparatory or decisional) processes [39]. The slope of the egocentric transformation was nearly flat $\left[y=11.63 x+1120.30, R^{2}=0.04\right]$ while for the object-based transformation it was notably more steep $\left[y=128.92 x+760.42, R^{2}=0.87\right]$. The intercept appears markedly higher in the egocentric group. However, although there was a tendency for response times to stimuli presented at $0^{\circ}$ to differ between transformations it did not reach Bonferroni corrected significance $[F(1,16)=4.03, p=0.062]$ and all other orientations did not statistically differ (all $p>0.370$ ). 

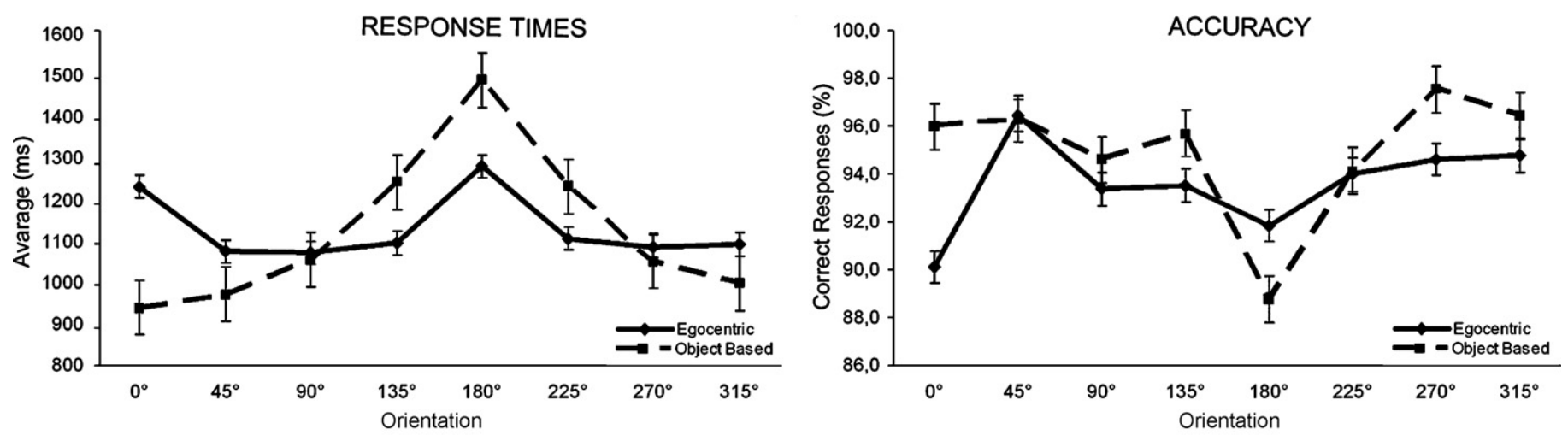

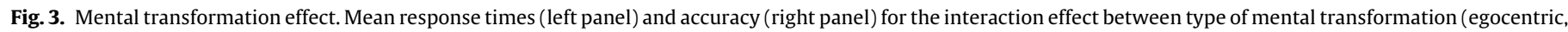
object-based) and stimuli orientation. Error bars depicts the standard error of the mean.

The stimulus laterality by orientation interaction was also significant $\left[F(7,112)=4.09, p<0.000\right.$ with $\left.\tilde{e}=0.89, \eta_{\mathrm{p}}{ }^{2}=0.20\right]$, showing the typical profile of response times for mental rotation of both "left" and "right" stimuli. Three simple main effects were significant with Bonferroni correction $(p \leq 0.005)$. Response time for "left" stimuli varied with orientation $[F(7,119)=14.48, p<0.000$ with $\left.\hat{\mathrm{e}}=0.31 \eta^{2}=0.46\right]$, as did response time for "right" stimuli $\left[F(7,119)=9.92, p<0.000\right.$ with $\left.\hat{\mathrm{e}}=0.29, \eta^{2}=0.37\right]$. In addition, response time for stimuli rotated at $180^{\circ}[F(1,17)=12.17, p=0.003$, $\left.\eta^{2}=0.42\right]$ were significantly slower for left $(1431 \mathrm{~ms})$ than for right $(1339 \mathrm{~ms})$ stimuli. Very nearly significant at the corrected alpha level, stimuli rotated to $45^{\circ}\left[F(1,17)=9.98, p=0.006, \eta^{2}=0.37\right]$ and $135^{\circ}\left[F(1,17)=9.63, p=0.006, \eta^{2}=0.36\right]$ also induced slower responses for left $\left(45^{\circ}, 1059 \mathrm{~ms} ; 135^{\circ}, 1201 \mathrm{~ms}\right)$ compared to right (987 ms; $1137 \mathrm{~ms})$ stimuli. And while stimuli presented at $0^{\circ}\left[F(1,17)=4.69, p=0.045, \eta^{2}=0.22\right]$ and at $90^{\circ}[F(1,17)=7.97$, $\left.p=0.012, \eta^{2}=0.32\right]$ clearly suggested a tendency toward significance (left: $0^{\circ}, 1114 \mathrm{~ms} ; 90^{\circ}, 1090 \mathrm{~ms}$; right: $1056 \mathrm{~ms} ; 1037 \mathrm{~ms}$ ), the remaining orientations depicting counter-clockwise or lateral rotations $\left(225^{\circ}, 270^{\circ}\right.$ and $\left.315^{\circ}\right)$ did not show any left-right difference (all $p \geq 0.403$ ).

\subsection{Response accuracy}

Response accuracy approached ceiling. Data are broken down by factor in Table 3 (supplementary materials). The ANOVA showed a significant main effect of stimulus orientation $[F(7,112)=4.85$, $p<0.000$ with $\left.\tilde{e}=0.77, \eta_{\mathrm{p}}{ }^{2}=0.23\right]$ and three significant interactions. Follow-up analyses of the three-way interaction of transformation by head constraint by stimulus laterality $[F(1,16)=5.62$, $\left.p=0.031, \eta_{\mathrm{p}}{ }^{2}=0.26\right]$ were non-significant and will not be described. The stimulus orientation effect was accounted for by the lowest accuracy at $180^{\circ}(90 \%)$ compared to all other orientations. The main effect of head constraint did not reach significance $[F(1,16)=1.7, p=0.21]$. The interaction of transformation with orientation $\left[F(7,112)=2.11, p=0.049, \eta_{\mathrm{p}}{ }^{2}=0.12\right]$ is illustrated in Fig. 3 (right panel). The simple main effect of the object-based transformation was approximately significant at the Bonferroni corrected level (0.005) after a violation of sphericity was accounted for $\left[F(7,56)=4.336, p=0.006\right.$ with $\left.\tilde{\mathrm{e}}=0.58, \eta^{2}=0.35\right]$. As was found with response time, the simple main effect of the egocentric transformation failed to reach corrected significance $[p=0.027$ with $\tilde{\mathrm{e}}=1.0$ ]. There was a tendency toward a significant difference between transformations at $0^{\circ}(p=0.019)$ and $270^{\circ}(p=0.066)$, while all remaining orientations indicated no difference in response accuracy for the two transformations (all $p \geq 0.194$ ).

A second two-way interaction was found between stimulus laterality and orientation $[F(7,112)=2.88 ; p=0.008$ with $\tilde{\mathrm{e}}=1.00$, $\left.\eta_{\mathrm{p}}{ }^{2}=0.15\right]$. Two simple main effects were significant at the Bonferroni corrected level (0.005). Response accuracy for "left" stimuli varied with orientation $[F(7,119)=5.05, p<0.003$ with $\hat{\mathrm{e}}=0.45$, $\eta^{2}=0.23$ ]; by contrast, accuracy for "right" stimuli failed to reach corrected significance $(p=0.024$ with $\tilde{\mathrm{e}}=0.90)$. Accuracy for left and right stimuli at most orientations varied by only $1-2 \%$. However, stimuli at $90^{\circ}$ did vary $\left[F(1,17)=11.85, p<0.003, \eta^{2}=0.41\right]$ with accuracy higher for right side stimuli (96\%) than left (92\%), and there was a tendency at $180^{\circ}$ for right side stimuli (92\%) to be more accurate than left $(88 \%)\left[F(1,17)=4.18, p=0.057, \eta^{2}=0.20\right]$.

\section{Discussion}

This study showed that response profiles for mental rotation of faces and faces-like stimuli were strongly influenced by the type of spatial transformation used, but not by the type of stimuli processed. Specifically, regardless of whether the stimulus was a human face, a non-human (owl) face, or a non-corporeal object (car), response times and accuracy of participants who used objectbased transformations were mediated by the degree of angular rotation, an effect notably absent in participants who used egocentric transformations.

Determining what has occurred in mental imagery studies is, at times, difficult given that imagery is a covert mental process and that different researchers may use different labels for similar processes. We have attempted to deal with the first issue through the use of the semi-structured questionnaire to elicit feedback about the strategy used. To deal with the second issue, we have adopted the operational definitions described in the comprehensive review of Zacks and Michelon [47]. Hence, we use "egocentric transformation" to mean the localization of an object to one's own frame of reference, and "object-based transformation" to refer to the localization of an object relative to a complex of axes relative to the same object.

In the present study, the analysis of the questionnaires administered after each block indicated that object-based imagers described the rotation on the vertical plane for all stimuli orientations, while egocentric imagers described several mental spatial transformations of themselves. This indicates that one set of instructions spontaneously lead to two entirely different strategies, and highlights the importance of recognizing the often glossed over role of individual differences in cognitive strategy and motor imagery performance [47]. Notably, and in accord to other studies which included the spatial transformation of one self with respect to a model room [34] or the spatial transformation of a car and of one's own perspective [45], the typical psychophysical profile for response times (mental rotation function) was found for the objectbased group, but not the egocentric group. This adds support to 
the idea of different cognitive strategies underlying the two mental transformations $[36,40,46]$. It might be argued that even in the case of an egocentric mental spatial transformation there could be an effect of the stimuli's orientation. However, the lack of such stimuli's orientation effect in the egocentric group can be interpreted as evidence of greater flexibility from physical laws [46]. In particular it has been shown that RTs for imagined egocentric transformations can be less dependent on the angle of rotation with respect to object-based mental transformations $[8,9,44,45]$. Neurophysiologically, egocentric imagery seems to cause a direct mapping into one's own body schema and involves overall motor processes, while object-based imagery relies less on motor processes [36,28]. Galati et al. [20], for example, demonstrated that in a visuospatial judgement task (line bisection) both strategies activate a fronto-parietal circuit, but that the object-based (labelled "allocentric" in the paper) strategy differed in that it had smaller activations throughout the circuit and additionally recruited subcortical regions related to spatial memory (hippocampal and parahippocampal). Similar findings have been reported with virtual reality navigation tasks [27], where the object-based ("allocentric") strategy additionally recruited the thalamus and cerebellum. Moreover, an object-based strategy is not completely independent from posture variations, suggesting that smaller motor activations are always needed to operate with body parts mental images [40].

Constraining head movement did not influence performance during the mental rotation of human faces. This result is the opposite of what is found for the mental rotation of other body parts, particularly hands. People tend to use egocentric transformations when hands are mentally rotated $[7,30,38]$, recruiting motor processes and structures in the process [11]. Subjects imagine moving their own hands from their actual posture into that of the stimulus for comparison [31], and thus the position of subject's own hands plays an important role in the ability to mentally manipulate hands in space $[11,33,40]$. Indeed, the effect of postural constraints is body part specific in that placing one's hands behind the back impairs the mental rotation of hands but not of feet [25]. Somewhat surprisingly, our results indicate that the physical constraint of head movement is a manipulation which does not influence performance during the mental rotation of faces. Previous work from our lab on cervical dystonia [18] provides some possible insights into this finding; for the healthy subjects included as a control group, the response times (combined left and right) for mental rotation of faces, hands, feet and cars showed that the slopes and intercepts for cars and faces were very similar and lower than for hands and feet. This suggests that mental rotation of body parts (i.e., hands and feet) and mental rotation of faces and faces-like stimuli are based, at least in part, on different processes. Moreover, Fiorio et al. [18] reported that patients with idiopathic cervical dystonia have a body-specific deficit in mental rotation in that all body parts are affected (head, hands, and feet). In contrast, patients with focalhand dystonia are impaired in the mental rotation of only hands but not feet, manifesting a representational deficit specific to the body part affected by the disease [17]. Hence, the lack of an effect of head constraint on mental rotation may be because the manipulation was applied to the vestibular and neck proprioceptive system which, as seen in cervical dystonia patients, is relatively robust to interference.

Another finding was that all three kinds of stimuli were processed in the same way. The face processing literature may be of help in understanding this counter-intuitive result. In particular, the face inversion effect indicates disproportionate disruption of faces recognition following their upside-down inversion, with respect to the recognition of other objects [4]. Interestingly it is also possible to "spread" the face inversion effect also to other nonface stimuli $[10,14]$. Indeed non-face objects as well as bodies [35] can be processed as faces $[14,42]$. It is worth noting that not only the animal face but also the front of the car used in the present study bears a structural resemblance with face stimuli.

The typical mental rotation function, that is the psychophysical profile showing the increase of response times as a function of the stimulus rotation, was preserved for both left and right stimuli. However comparing the performances for left and right stimuli rotated of the same degree, the stimuli which presented the black patch on the right side were identified in most of the cases faster than the stimuli that had the patch on the left side. It is important to note that most studies on mental rotation, including the present one, recruited only right-handed subjects and found faster responses for right stimuli with respect to left ones both in healthy participants $[19,22,25,30]$, and clinical patients [3,29]. The righthanders' preference for right stimuli is also consistent with a recent study that systematically investigated the preference for the "right" stimuli, demonstrating that the effect of laterality is preserved for right-handers (all subjects in the current study were right-handed) but not left-handers [26]. This might be explained by psychophysical evidence that left- and right-handed people have different representation of their own dominant hand [22], and that several factors, such as proprioception, handedness and visual familiarity, can orchestrate together in mental rotation [26]. In this way, we propose that faster and better responses for "right" stimuli recorded in the present study, is due to subject's hand dominance which is mirrored in their performance in mental rotation.

\section{Concluding remarks}

Subject's descriptions of cognitive strategy highlight that interindividual differences can lead to the spontaneous use of different imagery transformations in the presence of one set of instructions. This idea is in line with evidence that a mental strategy usually used for body parts can be used to mentally spatially transform an object [28]. Moreover, given that within each strategy all three types of face or face-like stimuli appear to have been processed in the same way, regardless of the possibility to move the head, we propose the data points to the dominance of the high-level process (mental transformation strategy) over the low-level one (elaboration of the stimulus), following a top-down pathway. This notion is in keeping with evidence that it is possible to voluntary adopt an egocentric (internal) or object-based (external) mental transformation to perform the same imagery task, resulting in the activation of two different but partially overlapping brain networks $[28,36]$ and supporting the dissociation between egocentric and object-based reference frame [18].

\section{Appendix A. Supplementary data}

Supplementary data associated with this article can be found, in the online version, at doi:10.1016/j.bbr.2009.10.037.

\section{References}

[1] Bakker M, de Lange FP, Stevens JA, Toni I, Bloem BR. Motor imagery of gait: a quantitative approach. Experimental Brain Research 2007;179(3):497-504.

[2] Briggs GG, Nebes RD. Patterns of hand preference in a student population. Cortex 1975;11(3):230-8

[3] Brugger P, Kollias SS, Muri RM, Crelier G, Hepp-Reymond MC, Regard M. Beyond re-membering: phantom sensations of congenitally absent limbs. Proceedings of the National Academy of Sciences USA 2000;97(11):6167-72.

[4] Carey S. Becoming a face expert. Philosophical Transactions of the Royal Society of London B: Biological Sciences 1992;335:95-103.

[5] Carpenter M, Proffitt DR. Comparing viewer and array mental rotations in different planes. Memory and Cognition 2001;29:441-8.

[6] Conson M, Sacco S, Sara M, Pistoia F, Grossi D, Trojano L. Selective motor imagery defect in patients with locked-in syndrome. Neuropsychologia 2008;46(11):2622-8.

[7] Cooper LA, Shepard RN. Mental transformations in the identification of left and right hands. Journal of Experimental Psychology: Human Perception and Performance 1975;104(1):48-56. 
[8] Creem SH, Wraga M, Proffitt DR. Imagining physically impossible transformations: geometry is more important than gravity. Cognition 2001;81:41-61.

[9] Creem-Regehr SH, Neil JA, Yeh HJ. Neural correlates of two imagined egocentric transformations. Neuroimage 2007;35(2):916-27.

[10] de Gelder B, Rouw R. Paradoxical configuration effects for faces and objects in prosopagnosia. Neuropsychologia 2000;38(9):1271-9.

[11] de Lange FP, Helmich RC, Toni I. Posture influences motor imagery: an fMRI study. Neuroimage 2006;33(2):609-17.

[12] Decety J, Jeannerod M, Germain M, Pastene J. Vegetative response during imagined movement is proportional to mental effort. Behavioral Brain Research $1991 ; 42(1): 1-5$

[13] Decety J, Jeannerod M, Prablanc C. The timing of mentally represented actions. Behavioral Brain Research 1989;34(1-2):35-42.

[14] Diamond R, Carey S. Why faces are and are not special: an effect of expertise. Journal of Experimental Psychology: General 1986;115(2):107-17.

[15] Dominey P, Decety J, Broussolle E, Chazot G, Jeannerod M. Motor imagery of a lateralized sequential task is asymmetrically slowed in hemi-Parkinson's patients. Neuropsychologia 1995;33(6):727-41.

[16] Fadiga L, Fogassi L, Pavesi G, Rizzolatti G. Motor facilitation during action observation: a magnetic stimulation study. Journal of Neurophysiology 1995;73(6):2608-11.

[17] Fiorio M, Tinazzi M, Aglioti SM. Selective impairment of hand mental rotation in patients with focal hand dystonia. Brain 2006;129:47-54.

[18] Fiorio M, Tinazzi M, Ionta S, Fiaschi A, Moretto G, Edwards MJ. Mental rotation of body parts and non-corporeal objects in patients with idiopathic cervical dystonia. Neuropsychologia 2007;45(10):2346-54.

[19] Funk M, Brugger P. Mental rotation of congenitally absent hands. Journal of International Neuropsychological Society 2008;14(1):81-9.

[20] Galati G, Lobel E, Vallar G, Berthoz A, Pizzamiglio L, Le Bihan D. The neural basis of egocentric and allocentric coding of space in humans: a functional magnetic resonance study. Experimental Brain Research 2000;133(2):156-64.

[21] Ganis G, Keenan JP, Kosslyn SM, Pascual-Leone A. Transcranial magnetic stimulation of primary motor cortex affects mental rotation. Cerebral Cortex 2000;10(2):175-80.

[22] Gentilucci M, Daprati E, Gangitano M. Right-handers and left-handers have different representations of their own hand. Brain Research Cognitive Brain Research 1998;6(3):185-92.

[23] Grezes J, Decety J. Functional anatomy of execution, mental simulation, observation, and verb generation of actions: a meta-analysis. Human Brain Mapping 2001;12(1):1-19.

[24] Harris IM, Harris JA, Caine D. Mental-rotation deficits following damage to the right basal ganglia. Neuropsychology 2002;16(4):524-37.

[25] Ionta S, Fourkas AD, Fiorio M, Aglioti SM. The influence of hands posture on mental rotation of hands and feet. Experimental Brain Research 2007;183(1):1-7.

[26] Ionta S, Blanke O. Differential influence of hands posture on mental rotation of hands and feet in left- and right-handers. Experimental Brain Research 2009;195(2):207-17.

[27] Jordan K, Schadow J, Wuestenberg T, Heinze HJ, Jancke L. Different cortical activations for subjects using allocentric or egocentric strategies in a virtual navigation task. Neuroreport 2004;15(1):135-40.
[28] Kosslyn SM, Ganis G, Thompson WL. Neural foundations of imagery. Nature Reviews Neuroscience 2001;2(9):635-42.

[29] Nico D, Daprati E, Rigal F, Parsons L, Sirigu A. Left and right hand recognition in upper limb amputees. Brain 2004;127(Pt 1):120-32.

[30] Parsons LM. Imagined spatial transformation of one's body. Journal of Experimental Psychology: General 1987;116(2):172-91.

[31] Parsons LM. Temporal and kinematic properties of motor behavior reflected in mentally simulated action. Journal of Experimental Psychology: Human Perception and Performance 1994;20(4):709-30.

[32] Parsons LM, Fox PT, Downs JH, Glass T, Hirsch TB, Martin CC. Use of implicit motor imagery for visual shape discrimination as revealed by PET. Nature 1995;375(6526):54-8.

[33] Petit LS, Pegna AJ, Mayer E, Hauert CA. Representation of anatomical constraints in motor imagery: mental rotation of a body segment. Brain and Cognition 2003;51(1):95-101.

[34] Presson CC. Strategies in spatial reasoning. Journal of Experimental Psychology: Learning, Memory and Cognition 1982;8:243-51.

[35] Reed CL, Stone VE, Bozova S, Tanaka J. The body-inversion effect. Psychological Science 2003;14(4):302-8.

[36] Ruby P, Decety J. Effect of subjective perspective taking during simulation of action: a PET investigation of agency. Nature Neuroscience 2001;4(5):546-50.

[37] Schwoebel J, Friedman R, Duda N, Coslett HB. Pain and the body schema: evidence for peripheral effects on mental representations of movement. Brain 2001;124(Pt 10):2098-104.

[38] Sekiyama K. Kinesthetic aspects of mental representations in the identification of left and right hands. Perception and Psychophysics 1982;32(2):89-95.

[39] Shepard RN, Metzler J. Mental rotation of three-dimensional objects. Science 1971;171(972):701-3.

[40] Sirigu A, Duhamel JR. Motor and visual imagery as two complementary but neurally dissociable mental processes. Journal of Cognitive Neuroscience 2001;13(7):910-9.

[41] Sirigu A, Duhamel JR, Cohen L, Pillon B, Dubois B, Agid Y. The mental representation of hand movements after parietal cortex damage. Science 1996;273(5281):1564-8.

[42] Tanaka JK, Gauthier I. Expertise in object and face recognition. In: Goldstone RL, Schyns PG, Medin DL, editors. Physiology of learning and motivation. Perceptual mechanisms of learning, vol. 36. San Diego: Academic Press; 1997. p. 83125.

[43] Tomasino B, Toraldo A, Rumiati RI. Dissociation between the mental rotation of visual images and motor images in unilateral brain-damaged patients. Brain and Cognition 2003;51(3):368-71.

[44] Wraga M. Thinking outside the body: an advantage for spatial updating during imagined versus physical self-rotation. Journal of Experimental Psychology: Learning, Memory, and Cognition 2003;29:993-1005.

[45] Wraga M, Creem SH, Proffitt DR. Updating displays after imagined object and viewer rotations. Journal of Experimental Psychology: Learning, Memory and Cognition 2000;26(1):151-68.

[46] Wraga M, Shephard JM, Church JA, Inati S, Kosslyn SM. Imagined rotations of self versus objects: an fMRI study. Neuropsychologia 2005;43(9):1351-61.

[47] Zacks JM, Michelon P. Transformations of visuospatial images. Behavioral and Cognitive Neuroscience Reviews 2005;4(2):96-118. 